Original Research Paper

\title{
Mutual Dynamics of Certain Types of Bitcoin: Data from Wavelet Coherence
}

\section{Vyacheslav Lyashenko ${ }^{1}$, Oleksandr Bilotserkivskyi ${ }^{2}$, Iryna Pyvavar ${ }^{3}$, Hryhorii Kots ${ }^{4}$}

${ }^{1}$ Department of Media Systems and Technology, Kharkiv National University of Radio Electronics, Ukraine.

${ }^{2}$ Department of Business, Trade and Products Expertise, National Technical University «Kharkiv Polytechnic Institute», Ukraine.

${ }^{3}$ Department of Economic Theory and Economic Policy, Simon Kuznets Kharkiv National University of Economics, Ukraine.

${ }^{4}$ Department of Cybersecurity and Information Technology, Simon Kuznets Kharkiv National University of Economics, Ukraine.

Article History

Received:

03.08.2021

Revised:

22.08.2021

Accepted:

26.08.2021

*Corresponding Author:

Vyacheslav Lyashenko

Email:

lyashenko.vyacheslav@gmail.com

This is an open access article, licensed under: $\mathrm{CC}-\mathrm{BY}-\mathrm{SA}$
Abstract: Unconventional methods of analysis are a good tool for studying new trends and phenomena. One of these objects of research is the cryptocurrency market. The paper shows the possibility and feasibility of applying the ideology of wavelets to analyze the dynamics of prices for cryptocurrencies. For this, the methodology of wavelet coherence is used. This methodology has been applied to various cryptocurrency pairs. The calculation results for real data are presented. It is shown that the results obtained agree with the theoretical conclusions and research results of other authors.

Keywords: Binance Coin, Bitcoin, Ethereum, Tether, Wavelet Coherence. 


\section{Introduction}

Statistical analysis is one of the elements for conducting research and describing the relationships between data that change over time [1] [2] [3] [4]. Such a study allows you to analyze the dynamics of data, reveal their relationship, and identify trends in data change. Another important point is the retrospective analysis of data trends [5]. This allows you to assess current trends, predict possible changes in the future. Thus, statistical analysis is an effective tool for data exploration.

Among such data can but any indicator sets. In this particular interest are data which have time dependence. It is the change over time that makes it possible to assess the dynamics of the data. This data can be from any field of study. Of particular interest are data that describe economic dynamics [3]. This is due to the fact that economic dynamics affects all spheres of human life.

Economic dynamics may reflect the processes that take place in different areas of economic life. The generalization of such phenomena is the dynamics of financial processes. The display of financial processes is the indicators of the financial market, banking, changes in currency quotes, and so on. At the same time, one of the important indicators of financial processes is the analysis of the dynamics of the cost of Bitcoin [6]. This is due to the fact that Bitcoin can be considered both as a means of investment, and as a means of deposits, and as a means of accumulation. At the same time, different types of Bitcoins can be distinguished. Then a number of questions arise that relate to the analysis of the mutual relationships between the dynamics of different types of Bitcoins. It is these questions that are considered in this work.

\section{Materials and Methods}

\subsection{Brief Analysis of Related Work}

There are many works devoted to the study of the Bitcoin dynamics. These works look at both Bitcoin and its various types.

Bariviera, et al [7] examine various statistical properties of the Bitcoin market. First of all, the authors analyze the profitability of Bitcoin for different time periods. Such an analysis is carried out in Comparative aspect, the dynamics of different currencies. The authors also investigate the memory of the time series that are analyzed. However, such research is carried out only for Bitcoin - the main cryptocurrency.

The dynamics of the price process on the Bitcoin market is discussed in the article by Pichl \& Kaizoji [8]. This analysis is based on studying the dynamics of Bitcoin price volatility. To do this, the authors use standard statistical and technical analysis procedures. In such a study, only the dynamics of Bitcoin prices are analyzed.

The dependence of the dynamics of prices for Bitcoin on demand and supply is considered in the work of the Luis et al. [9]. For this, the classical demand model is used, which is based on various statistical estimates. The authors consider a regression analysis model and an error correction model. In this form, the authors investigate the long-term and short-term relationships between the variables from the statistical analysis - sales volumes, demand volumes, supply volumes, Bitcoin cost. This allows you to analyze the dynamics of Bitcoin prices for different time periods. But this does not take into account the dynamics of prices for other types of cryptocurrencies.

In his research, Kristoufek conducts a comparative analysis of the two Bitcoin markets - against the US dollar and the Chinese Yuan [10]. For this analysis, the author uses an efficiency index. The performance index indicators are based on fractal dimension analysis and entropy analysis. This allows for a detailed analysis of Bitcoin price dynamics.

Osterrieder \& Lorenz [11] analyze the levels of profitability of different types of Bitcoins. At the same time, the authors compare the yield of Bitcoins with the traditional exchange rates of the G10 currencies in relation to the dollar. For such an analysis, classical methods of statistical analysis are used, which allow one to study the distributions of such dynamics of profitability and their heavy tails. This allows you to better explore the properties of Bitcoin volatility and assess their dynamics.

In the work of the Grigoriev et al [12], a statistical analysis of price dynamics for various cryptocurrencies is carried out. In a comparative aspect, the authors investigate the dynamics of prices for various types of cryptocurrencies. At the same time, special attention is paid to the capitalization of such assets. This capitalization is valued in US dollars, which simplifies the analysis.

A brief analysis on the research topic shows that cryptocurrency price dynamics are used for the appropriate analysis. In this case, for such an analysis, various statistical methods are used. At the same time, one of the most promising methods is analysis based on the approaches of chaos theory and analysis of self-similar series. The approach based on the ideology of wavelets and which correlates 
with the approach based on the calculation of the Hurst exponent can be considered just as promising. It should also be noted that the ideology of wavelets is widely used to analyze data that is presented as a time series [5] [6] [13] [14]. Among the key approaches ideology of wavelets, one should single out the methodology of wavelet coherence.

\subsection{Wavelet Coherence as an Analysis Tool}

Wavelet coherence refers to methods that are based on the ideology of wavelets. Wavelet - can be written as a specialized function that allows you to highlight changes in time series data. The multiplicative combination of the time series and the wavelet function allows you to select the critical points of the time series that is being investigated. This can be done because the wavelet is a function rapidly decreasing at infinity, the average value of which is zero. Then, at the point where the values of the time series change, the fractal transitions are characterized by the finite value of the derivative. As a result, this allows you to highlight the areas of change in values for the time series.

If we have two time series, then we use wavelet coherence. Wavelet coherence is analogous to multiple correlations over time. Then we can estimate the level of dependence between the data in both the time and frequency planes.

To implement wavelet coherence, the concept of cross wavelet spectra is used. Suppose we have two series of data $f 1(t)$ and $f 2(t)$ then we can determine the wavelet coherence [5], [6], [15], [16]:

$$
R^{2}(a, b)=\frac{\left|G\left(a^{-1} W_{f 1 f 2}(a, b)\right)\right|^{2}}{G\left(a^{-1}\left|W_{f 1}(a, b)\right|^{2}\right) G\left(a^{-1}\left|W_{f 2}(a, b)\right|^{2}\right)},
$$

where:

$\mathrm{W}(\mathrm{a}, \mathrm{b}) \quad$ values of cross wavelet spectra

a determines the size of the wavelet and is called the scale,

b specifies the temporal localization of the wavelet and is called a shift (the abscissa is the wavelet shifts (time axis $-\mathrm{t}$ ), the ordinate is the scale; abscissa is the variable that displays the data number in the series under investigation and ordinate is the variable that displays the depth of cross-links for a time series);

$\mathrm{G} \quad$ is a smoothing operator;

$\mathrm{R}^{2}(\mathrm{a}, \mathrm{b}) \quad$ the squared wavelet coherency coefficient. $0 \leq \mathrm{R}^{2}(\mathrm{a}, \mathrm{b}) \leq 1$. If these values tend to zero, then we have a weak correlation. Otherwise, we have a strong correlation [5] [15].

As a result, we get some dependence in the two-dimensional plane. The abscissa shows changes in wavelet coherence $R^{2}(a, b)$ values over time. The ordinate shows changes in the wavelet coherence values, which take into account the depths of cross-references between time series $f 1(t)$ and $f 2(t)$. Such a graph also has a color grading that displays the level of wavelet coherence values.

\subsection{Cryptocurrency Types as Data for Analysis}

There are several types cryptocurrency, which generally are a variety of digital currency. Among the varieties of cryptocurrency, there are: Bitcoin, Altcoins and Tokens [17] [18]. Bitcoin is the first, basic cryptocurrency. Altcoins are new types of cryptocurrency that appeared after Bitcoin. Tokens are a derivative of cryptocurrencies. Tokens are a unit of account that is designed to represent the digital balance in a certain cryptocurrency asset [18] [19].

Below is the dynamics of some cryptocurrencies that we will use for analysis in this work. This selection is based on the market cap metric. We have selected the first four cryptocurrencies that have the largest market cap as of 03.08.21.

Among these cryptocurrencies, the following are selected: Bitcoin, Ethereum (a kind of Altcoins), Tether (a kind of Tokens) and Binance Coin (a kind of Tokens). For the analysis, we have chosen the period from 01.01.21 to 03.08.21. In this case, the choice of the period is not critical. It is important for us to show the possibility of using wavelet coherence for the corresponding analysis. All data from investing.com. 
In Figure 1 shows the dynamics of cryptocurrencies that we have chosen for analysis.

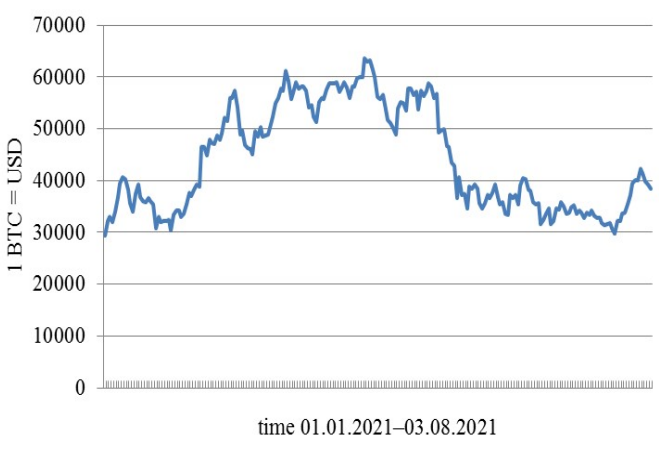

(a) Bitcoin

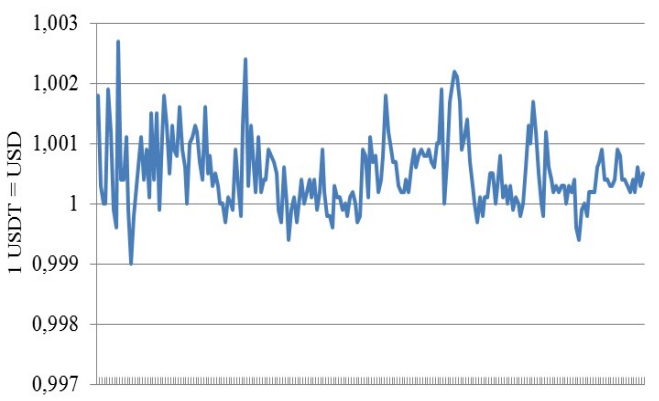

time $01.01 .2021-03.08 .2021$

(c) Tether

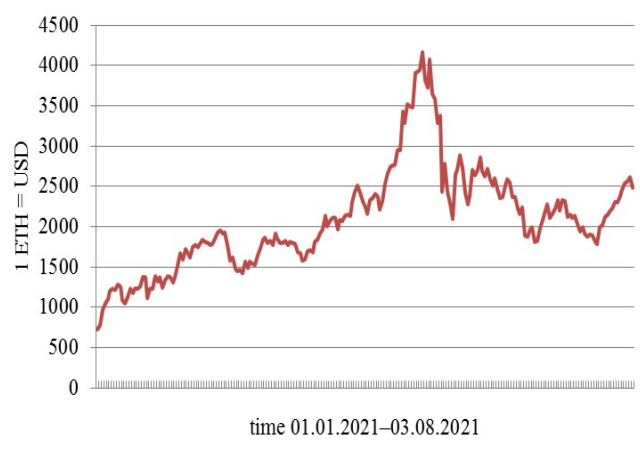

(b) Ethereum

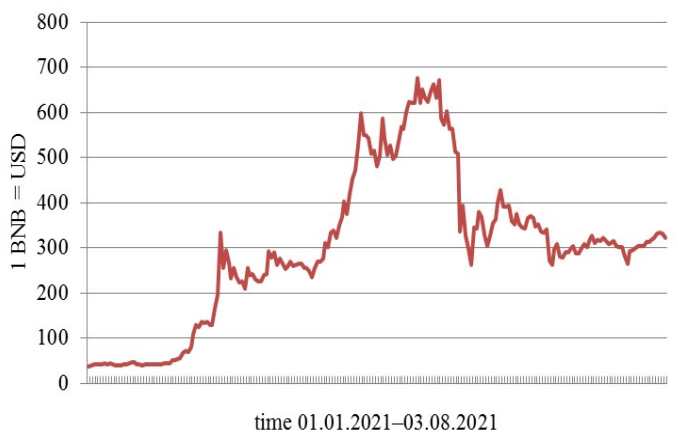

(d) Binance Coin

Figure 1. Dynamics of Prices for Some Cryptocurrencies

We see that the dynamics of prices for cryptocurrencies is different. Therefore, we will analyze how such dynamics relate to each other (for each pair of cryptocurrencies).

\section{Results and Discussion}

Figure 2 - Figure 7 shows the dynamics of the wavelet coherence values for each pair of cryptocurrencies.

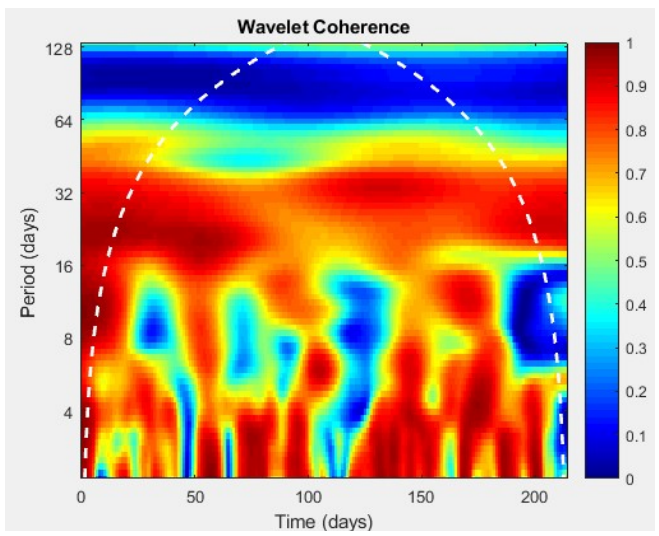

Figure 2. Wavelet Coherence between Data that Describe the Price Dynamics of Bitcoin and Ethereum 


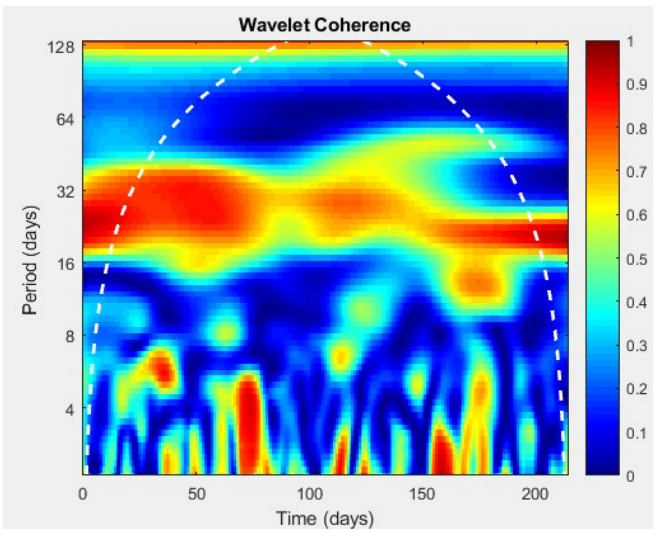

Figure 3. Wavelet Coherence between Data that Describe the Price Dynamics of Bitcoin and Tether

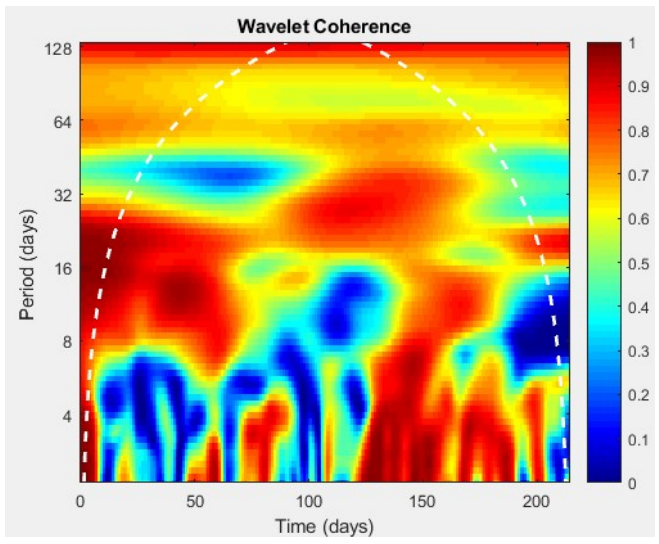

Figure 4. Wavelet Coherence between Data that Describe the Price Dynamics of Bitcoin and Binance Coin

In Figure 2 till Figure 7, we see the display of the wavelet coherence values in color. The significance of such a color scheme is presented in the form of a column, which is located to the right of the main graph. Figure 2 - Figure 4 also shows the region of the most reliable values of the wavelet coherence, which is limited by a dotted line in the form of a parabola.

We can observe some correlation between the data that describe the price dynamics of Bitcoin and Ethereum, Tether, Binance Coin, respectively (Figure 2, Figure 3 and Figure 4). The greatest consistency between the respective data series is observed for Bitcoin and Ethereum, Bitcoin and Binance Coin. Here we see consistency, both in the short term and long term. This can be explained by the fact that Bitcoin is the underlying cryptocurrency, and Binance Coin is a token that is meant for balance. The consistency between Bitcoin and Ethereum is explained by the fact that these are two types of major cryptocurrencies that have approximately the same trends in the development of their price dynamics (see Figure 1). This primarily applies to periods of growth and fall in prices for cryptocurrencies.

At the same time, the consistency between Bitcoin and Tether is significantly less. This suggests that Tether is a poor instrument for balancing digital assets over the time frame under consideration. 
Figure 5 and Figure 6 shows the wavelet coherence values between Ethereum and Tether, Ethereum and Binance Coin.

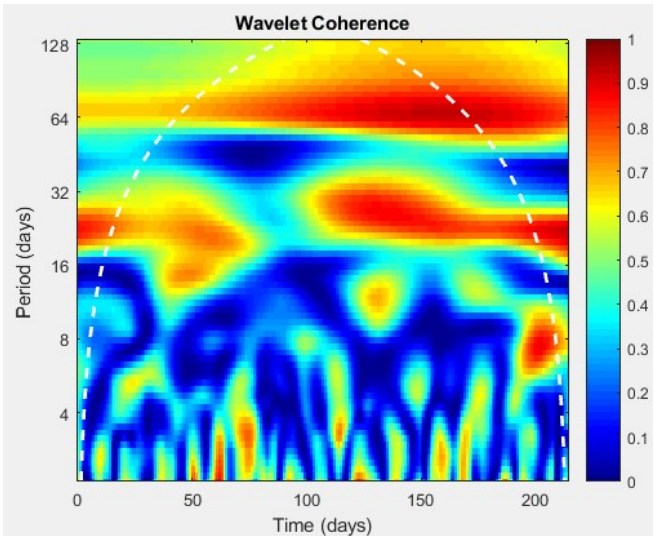

Figure 5. Wavelet Coherence between Data that Describe the Price Dynamics of Ethereum and Tether

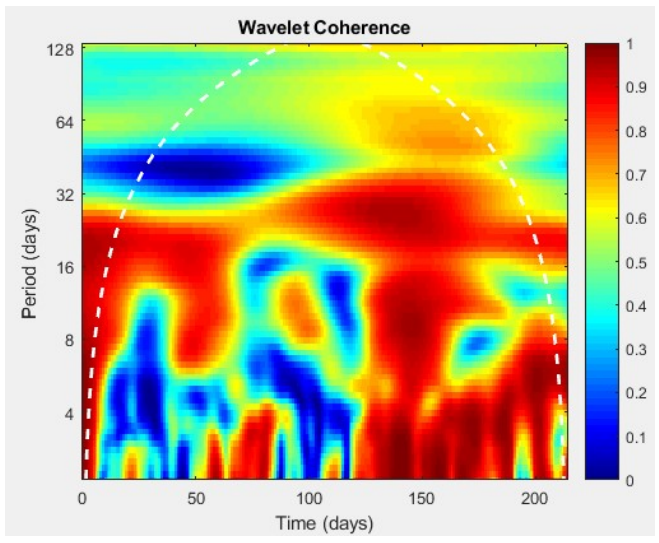

Figure 6. Wavelet Coherence between Data that Describe the Price Dynamics of Ethereum and Binance Coin

Figure 7 shows the wavelet coherence values between Tether and Binance Coin.

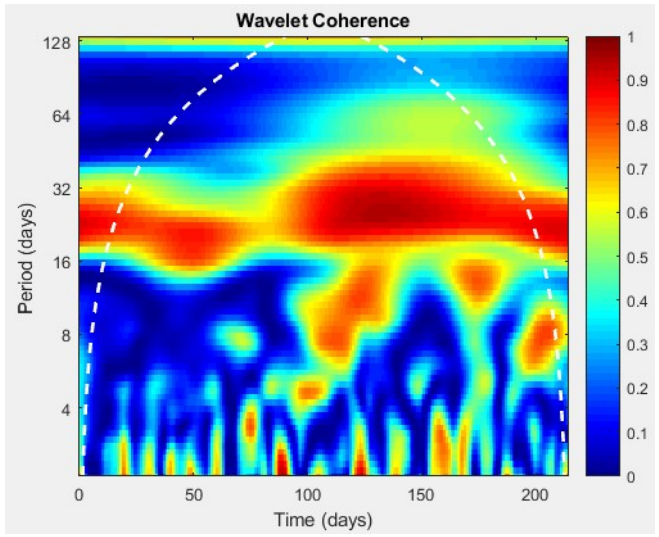


Figure 7. Wavelet Coherence between Data that Describe the Price Dynamics of Tether and Binance Coin

The data in Figure 5 till Figure 7 inherits the data in Figure 2, Figure 3 and Figure 4 but is less pronounced. This is because, that Bitcoin is the underlying cryptocurrency, unlike Ethereum. Nevertheless, the data in Figure 2 - Figure 7 do not contradict each other. Therefore, the wavelet analysis methodology can be used to study the joint price dynamics of various types of cryptocurrencies.

We can see that the consistency between Tether and Binance Coin is negligible (especially in the short term). This is due to the fact that these cryptocurrencies are used for different types of settlements and are differently backed by real currency [20]. However, over the long term, we are seeing consistency between Tether and Binance Coin. This is due to the nature of these cryptocurrencies, and also confirms the feasibility of using wavelet coherence for conducting relevant research.

At the same time, it should be noted that the considered relationships are confirmed by the studies of other authors. The work of Alexander et al [21] notes the importance of the relationship between Bitcoin and Binance Coin. At the same time, Wei emphasizes the connection between Bitcoin and Tether, pointing out the presence of a time lag in such a connection [22]. In our study, this manifests itself in longer-term consistency between the data. Therefore, the results presented above are reliable, and the research methods are appropriate for the appropriate analysis.

\section{Conclusion}

Modern trends in the development of the cryptocurrency market necessitate a deeper consideration of its development. For this, it is advisable to apply various methods of analysis and research. Based on this, we considered the possibility of applying the theory of wavelet analysis to analyze the dynamics of prices for cryptocurrencies. For this, we used the wavelet coherence methodology. We looked at various pairs of cryptocurrencies. The results obtained are consistent with theoretical conclusions about the dynamics of cryptocurrency prices. These data are also confirmed by the studies of other authors. The work presents real data and the results of their processing. This allows you to make informed decisions, choose the necessary strategies for the cryptocurrency market.

\section{References}

[1] A. Tromelin, C. Chabanet, K. Audouze, F. Koensgen, and E. Guichard, "Multivariate statistical analysis of a large odorants database aimed at revealing similarities and links between odorants and odors", Flavour and Fragrance Journal, vol. 33, no. 1, pp. 106-126, 2018. doi: $10.1002 /$ ffj. 3430 .

[2] M. Abdullahi, and K. Bala, K. "Analysis of the Causality Links between the Growth of the Construction Industry and the Growth of the Nigerian Economy", Journal of Construction in Developing Countries, vol. 23, no. 1, pp. 103-113, 2018. doi: 10.21315/jcdc2018.23.1.6.

[3] A. Kuzemin, and V. Lyashenko, "Analysis of Spatial-temporal Dynamics in the System of Economic Security of Different Subjects of Economic Management", International Journal Information Technologies and Knowledge, vol. 2, no. 3, pp. 234-238, 2008.

[4] I. Dobrovolskaya, and V. Lyashenko, "Interrelations of banking sectors of European economies as reflected in separate indicators of the dynamics of their cash flows influencing the formation of the resource potential of banks", European Applied Sciences, vol. 1-2, pp. 114-118, 2013.

[5] O. Vasiurenko, and V. Lyashenko, "Wavelet coherence as a tool for retrospective analysis of bank activities", Economy and Forecasting, vol. 2, pp. 43-60, 2020. doi: 10.15407/eip2020.02.043.

[6] N. Shelud'ko, O. Vasiurenko, V. Lyashenko, and N. Morozova, "Gold and Bitcoin Price Dynamics as a Reflection of Investor Sentiment”, Journal La Bisecoman, vol. 1, no. 4, pp. 1925, 2020. doi: 10.37899/journallabisecoman.v1i4.218.

[7] A. F. Bariviera, M. J. Basgall, W. Hasperué, and M. Naiouf, "Some stylized facts of the Bitcoin market", Physica A: Statistical Mechanics and its Applications, vol. 484, pp. 82-90, 2017. doi: 10.1016/j.physa.2017.04.159.

[8] L. Pichl, and T. Kaizoji, "Volatility analysis of bitcoin", Quantitative Finance and Economics, vol. 1, no 4, pp. 474-485, 2017. doi: 10.3934/QFE.2017.4.474. 
[9] P. Luis, G. de la Fuente, and J. Perote, "The drivers of Bitcoin demand: A short and long-run analysis", International Review of Financial Analysis, vol. 62, pp. 21-34, 2019. doi: 10.1016/j.irfa.2019.01.006.

[10] L. Kristoufek, "On Bitcoin markets (in) efficiency and its evolution", Physica A: statistical mechanics and its applications, vol. 503, pp. 257-262, 2018. doi: 10.1016/j.physa.2018.02.161.

[11] J. Osterrieder, and J. Lorenz, "A statistical risk assessment of Bitcoin and its extreme tail behavior", Annuals of Financial Economics, vol. 12, no. 01, pp. 1750003, 2017. doi: $10.1142 / \mathrm{S} 2010495217500038$.

[12] V. V. Grigoriev, A. Koshelev, and A Glisin, "Determining the Future Profitability of the Main Types of Cryptocurrencies", In Computer Science On-line Conference, pp. 171-181, 2021. doi: 10.1007/978-3-030-77448-6_15.

[13] N. Iqbal, Z. Fareed, F. Shahzad, X. He, U. Shahzad, and M. Lina, "The nexus between COVID19, temperature and exchange rate in Wuhan city: new findings from partial and multiple wavelet coherence", Science of The Total Environment, vol. 729, pp. 138916, 2020. doi: 10.1016/ j.scitotenv.2020.138916.

[14] S. Y. Choi, "Industry volatility and economic uncertainty due to the COVID-19 pandemic: Evidence from wavelet coherence analysis", Finance Research Letters, vol. 37, 101783, 2020. doi: $10.1016 /$ j.frl.2020.101783.

[15] C. Torrence, and P. J. Webster, "Interdecadal changes in the ENSO-monsoon system", Journal of climate, vol. 12, no. 8, pp. 2679-2690, 1999. doi: 10.1175/15200442(1999)012<2679: ICITEM>2.0.CO;2.

[16] O. Vasiurenko, V. Lyashenko, V. Baranova, and Z. Deineko, "Spatial-Temporal Analysis the Dynamics of Changes on the Foreign Exchange Market: an Empirical Estimates from Ukraine", Journal of Asian Multicultural Research for Economy and Management Study, vol. 1, no. 2, pp. 1-6. 2020. doi: 10.47616/jamrems.v1i2.41.

[17] M. P. Kucheryavenko, O. O. Dmytryk, and O. O. Golovashevych, "Cryptocurrencies: development, features and classification", Financial and credit activity: problems of theory and practice, vol. 3, no. 30, pp. 371-374, 2019. doi: 10.18371/fcaptp.v3i30.179737.

[18] A. Hnatovska, O. Manzhosova, A. Marushchak, and U. Oleksiy, "The Place of Cryptocurrency in the System of Financial Instruments", Amazonia Investiga, vol. 8, no. 24, pp. 260-266, 2019.

[19] K. F. Low, and E. G. Teo, "Bitcoins and other cryptocurrencies as property?", Law, Innovation and Technology, vol. 9, no. 2, pp. 235-268, 2017. doi: 10.1080/17579961.2017.1377915.

[20] Q. Ji, E. Bouri, L. Kristoufek, and B. Lucey, "Realised volatility connectedness among Bitcoin exchange markets", Finance Research Letters, pp. 101391, 2021. doi: 10.1016/j.frl.2019.101391.

[21] C. Alexander, D. Heck, and A. Kaeck, "The Role of Binance in Bitcoin Volatility Transmission", 2021. arXiv preprint arXiv:2107.00298.

[22] W. C. Wei, "The impact of Tether Grants on Bitcoin", Economics Letters, vol. 171, pp. 19-22, 2018. doi: 10.1016/j.econlet.2018.07.001. 